http://jmscr.igmpublication.org/home/ ISSN (e)-2347-176x ISSN (p) 2455-0450

crossref DOI: https://dx.doi.org/10.18535/jmscr/v8i1.167

Journal Of Medical Science And Clinical Research

\title{
Co-occurrence of Epistaxis in Migraine Sufferers - A Hospital Based Prospective Observational Study from Kerala
}

\author{
Authors \\ Dr Kabeer Kaithadiyil Abdul Khayar Kutty ${ }^{1}$, Dr Sunil Das ${ }^{2 *}$, Dr S.R .Chandra ${ }^{3}$, \\ Dr Jyothish Sivananda Panicker ${ }^{4}$, Dr Sreedevi Parameswara Panicker ${ }^{5}$ \\ ${ }^{1}$ Additional Professor, Department of Neurology, Government T.D. Medical College, Alappuzha \\ ${ }^{2}$ Assistant Professor, Department of Neurology, Government Medical College, Trivandrum \\ ${ }^{3}$ Professor, Department of Neurology, NIMHANS, Bangalore \\ ${ }^{4}$ Additional Professor, Department Of Neurosurgery, Medical College, Trivandrum \\ ${ }^{5}$ Consultant in Pediatrics, General Hospital, Alappuzha \\ *Corresponding Author \\ Dr Sunil Das \\ Assistant Professor, Department of Neurology, Government Medical College, Trivandrum, India
}

\begin{abstract}
Background: Migraine is a common type of primary head ache syndrome. Various co morbid conditions have been associated with migraine. This include stroke, epilepsy, psychiatric illness, fibromyalgia, restless leg syndrome etc.

We have observed that there is an increased incidence of epistaxis in patients admitted with acute migraine. Objectives: Present study aims is to determine the incidence of epistaxis in patients with migraine.

Materials and Methods: All patients who satisfied the criteria of migraine based on ICHD 2 were included in this study. Patients with features of secondary headache syndromes and other causes of epistaxis were excluded from the cohort using appropriate investigations. This is a hospital based prospective observational study conducted in two tertiary institutions of Kerala over a period of seven years.

Observations: Total number of patients enrolled for study was 486, of which 331 (68.1\%) were females and 155(31.9\%) were males. 433 patients (89.09\%) were between the age of 1land $45 y \mathrm{rs}, 418(86 \%)$ had one or more co morbid diseases and 117(24.07\%) had one or more recurrent episodes of epistaxis during acute phase of migraine. 452 patients (93\%) with epistaxis also had other associated illnesses.

Conclusions: Epistaxis is more frequently seen in migrainers than in general population. Awareness of cooccurrence of migraine and epistaxis is important to avoid unnecessary investigations and the use of NSAID during acute phase of illness.

Keyword: Migraine, Epistaxis, Co morbid illness.
\end{abstract}

\section{Introduction}

Migraine is one of the most common illnesses worldwide, affecting around twenty percent of population with significant morbidity. The term migraine was coined by Galen in 200AD, which means hemicranias in Greek. Migraine is a multifactorial disorder with genetics playing an important role in the susceptibility, and 
symptomatology, as well as comorbidity with other traits and conditions. There are four phases in migraine such as prodrome, aura, the headache phase and postdrome. Premonitory symptoms are likely to be more common than has been reported in the literature, and if patients are asked specifically about each symptom, many will report several symptoms in association with attacks. Symptoms reported can be broadly categorized into three separate groups: fatigue and cognitive changes, homeostatic alterations, and sensory sensitivities. Diagnosis of migraine is based on history and clinical examination. There is no definite imaging or serological marker available to confirm the diagnosis. Being a primary headache syndrome all possible secondary headache syndromes must be ruled out before making a diagnosis of migraine. Various associated symptoms like nausea, vomiting, photophobia, and photophobia may also help the clinician to make definite diagnosis of migraine. Diagnosis of migraine is done based on ICHD 2 criteria. Neural substrate for migraine is only poorly understood even after continuous research for more than twenty years. Even today we have to stick on to symptomatic therapy because we cannot rely upon any specific therapy targeting at molecular level. Investigation of the genetic factors involved in migraine have used, family studies for the rare Mendelian forms of migraine, as well as large genome-wide association studiesin case control cohorts for the common polygenic form of migraine, for gene discovery and further understanding of the pathways and basic biology of the disorder. There will be change in brain function of thalamo cortical circuit during premonitory phase of migraine as evidenced by electrophysiological as well as functional MRI studies. ${ }^{11}$ In different animal models, including non-human primates, activation of the meningeal trigeminovascular afferents, leads to activation of second-order dorsal horn neurons in the Trigeminal Nucleus pars Caudalis (TNC) and the two uppermost divisions of the cervical spinal cord. Perception of pain is made possible by impulses that travel anteriorly to brain areas involved in perception of pain, like periaqueductal gray matter and various thalamic nuclei.

Feinstein introduced the term comorbidity in 1970 to refer co-occurrence of two different disease entities in same person at a rate higher than we expected ${ }^{4}$. Comorbidity is clinically relevant due to various reasons:(1) Comorbid disease may mask or change the profile of clinical presentation (2)One disease may remind the treating physician about other co-occurring conditions (3) Clinician can choose a drug which is effective for both disease entities (4) Associated disease may provide a clue about the pathophysiology of migraine. Migraine may be a manifestation of various vascular diseases like cerebral autosomal dominant arteriopathy with subcortical infarcts and leukoencephalopathy, retinal vasculopathy with cerebral leukodystrophy and hereditary systemic angiopathy. A lot of neuropsychiatric conditions like generalized anxiety and panic attack may complicate the presentation of migraine according to clinical and community based studies. The relation between epilepsy, obesity and asthma with migraine is controversial. Several chronic pain disorders were reported to be associated with migraine. As migraine with aura is a well-documented risk factor for cerebrovascular accident it is important to investigate for other underlying risk factors in any person with this disease entity. In patients with history of cerebrovascular disease, tryptans and ergotamines must be avoided during acute migraine management. Try to avoid tricyclic antidepressants for prophylactic therapy.

Epistaxis is an important otolaryngological emergency and is defined as bleeding from nasal cavity and/or nasopharynx. Epistaxis may be classified as anterior or posterior. Epistaxis can be due to various local or systemic causes. Trauma due to nose picking and bleeding from Kiesselbach plexus constitute most common local causes ${ }^{9}$. Hypertension, coagulopathies, neoplasms, use of nasal steroids, vascular anomalies, upper respiratory infections, treatment with antiplatelet 
and anticoagulants are to be considered in any patient with epistaxis. According to the once widely accepted 'Vascular Theory of Migraine', the symptoms of migraine aura are caused by transient ischemia that is induced by vasoconstriction, and the headache arises from rebound abnormal vasodilation of intracranial arteries and consequent mechanical activation of perivascular sensory fibres $1^{2}$. Epistaxis is an unrecognized illness coexisting with acute migraines, with limited literature backup ${ }^{10}$.

\section{Aim and Objective of Study}

Aim of the study is to assess the association between Migraine and Epistaxis. Objective of the study is to evaluate the incidence of epistaxis in patients with migraine, especially during an acute attack.

\section{Materials and Methods}

This prospective observational study was conducted in department of Neurology of two medical colleges in Kerala over a period of seven years.

\section{Inclusion Criteria}

Any patient between age of ten and sixty five years satisfying the ICHD II R1 criteria of migraine were included for this study.

\section{Exclusion Criteria}

1. Any cause of secondary headache syndrome.

2. Any local cause for epistaxis.

3. History of treatment with antiplatelet and anticoagulant.

4. Any coagulation abnormality.

5. History of trauma /systemic and central nervous system malignancy/infections.

6. History of systemic hypertension.

Study was conducted at two tertiary centers in Kerala over a period of seven Years. Baseline demographic data were recorded and a detailed neurological examination was done in all patients. Common causes of epistaxis were excluded using appropriate laboratory investigations including bleeding time, clotting time, APTT, prothrombin time, platelet count and otorhinological examination (to rule out local vascular anomalies). Secondary headache was ruled out using Brain imaging (Contrast CT scan brain/MRI Brain \& MR Venogram)

\section{Observations}

At the end of study period results were analyzed systematically. Of the cohort of 486 subjects $331(68.1 \%)$ were females and 155 (31.9\%) were males. 433 patients ( $89.09 \%$ ) were between the age of 11 and 45.418 patients $(86 \%)$ had one or more co morbid diseases. Migraine with visual aura was noticed in 83 patients $(17.07 \%)$ and migraine with brain stem aura in 34 (6.99\%). 83 (17.07\%) had diabetes mellitus ,fibromyalgia was in $117(24.07 \%)$ and depression in 151 $(31.06 \%)$.Other observations include anxiety disorders in 156 (32.09\%), hypothyroidism in 107 $(22.01 \%)$, carpal tunnel syndrome in $165(33.95 \%)$, seronegative spondylo arthropathy in $58(11.93 \%)$, peripheral vertigo in 155 $(31.89 \%)$, dyslipidemia in $82(16.87 \%)$ bronchial asthma in 54(11.11\%) obesity in 92(18.93\%), low back pain in 184(37.86 \%). Hypertensive patients were excluded from the study. In this study 117 (24.07\%) patients had one episode of epistaxis during acute migraine and 6 percent had repeated episodes of epistaxis.

Table 1.Age and sex distribution of patients with migraine

\begin{tabular}{|l|c|c|}
\hline Sex & Total number & Percentage \\
\hline Male & 331 & 68.1 \\
\hline Female & 155 & 31.9 \\
\hline
\end{tabular}




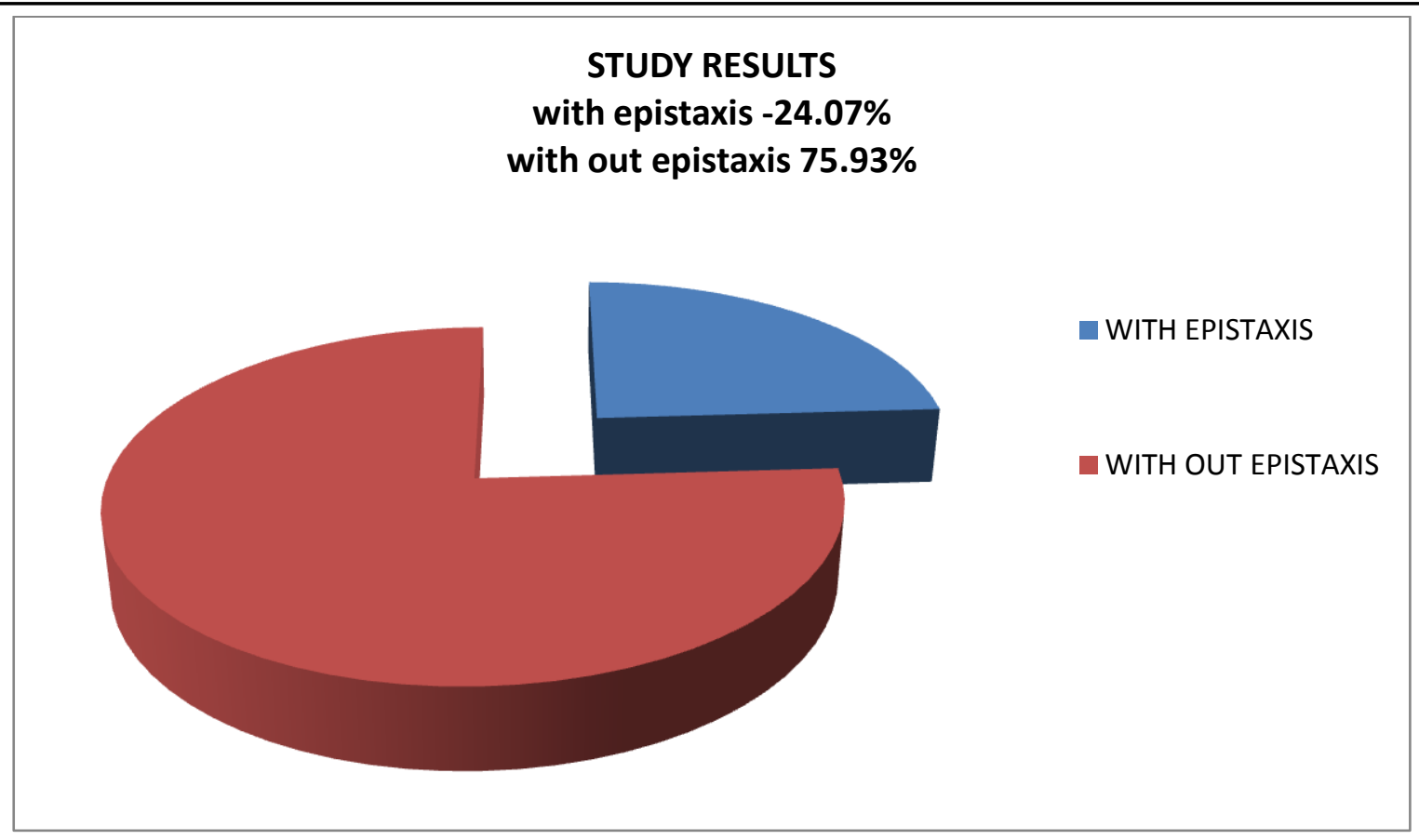

Figure 1.Comparison of patients with or without epistaxis

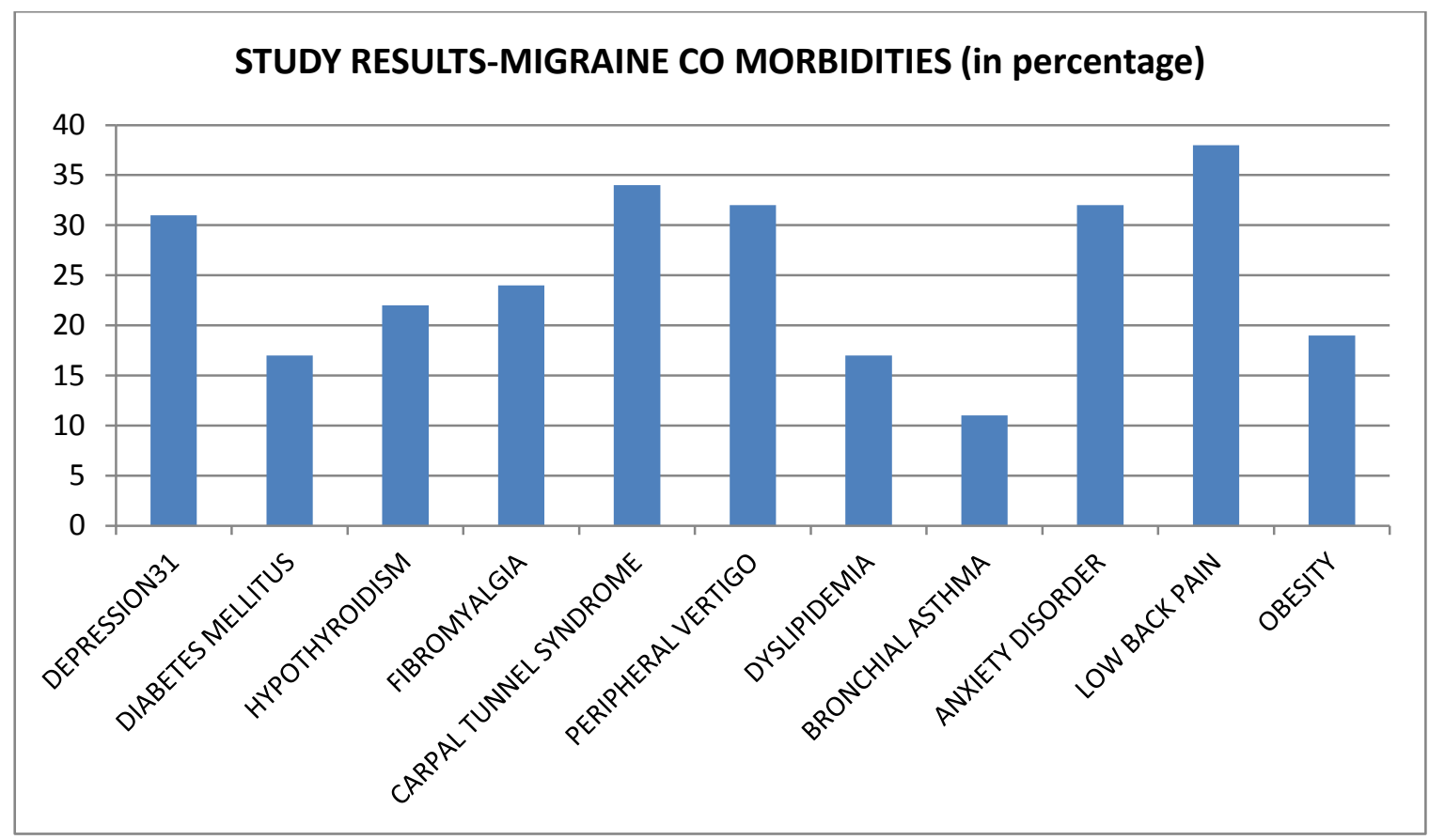

Figure 2 Distribution of co morbidities in migraine sufferers

\section{Discussion}

Within the skull, pain sensitivity is primarily restricted to the meningeal blood vessels, which are densely innervated by nociceptive sensory afferent fibres of the ophthalmic division of the trigeminal nerve ${ }^{8}$. It is generally recognized that the development of migraine headache depends on the activation of these afferents. Epistaxis during a migraine attack could probably be explained by a common origin, trigeminovascular system (TVS) activation. Nostril irrigation depends on external and internal carotid artery terminal branches, which coalesce and form an arterial area that relies on the trigemino vascular system The main blood supply to nostrils is from both external carotid (internal maxillary artery) and the internal carotid arteries (ethmoidal arteries, branches of ophthalmic artery). Trigemino vascular system 
activation would provoke vasodilatation and subsequent bleeding. Several facts support this theory ${ }^{6}$. Trigeminal activation increasing extra cerebral flow has been proven and the implications of this system in the physiopathology of migraine are not in dispute ${ }^{7}$. Furthermore, the presence of facial flushing and vasodilation of extracranial arteries prior to pain, have been described in migraine along with their appearance in other trigeminal cephalalgias, such as cluster headache. Although the possibility of epistaxis during migraine attacks is infrequent, it is possible that both conditions are due to TVS activation.

\section{Conclusions}

Present study confirmed that there is definite cooccurrence of epistaxis in migrainers compared general population. On searching the literature we could obtain only scanty information about this rare association. Epistaxis is a frightening and potentially life threatening medical emergency and most of the time we could not identify any underlying cause for bleeding. A lot of the laboratory tests will yield negative results and the treating physician may be forced to send unnecessary invasive or noninvasive investigations in a desperate attempt to find out a cause and to prevent recurrence. The awareness of this association between two disease entities will save time ,avoid excessive anxiety and ensure prompt therapy during the critical phase of the illness. In almost all patients recurrence of epistaxis can be abolished, by administration of appropriate prophylactic treatment for migraine. But there is no consensus regarding the duration of medication in order to prevent delayed occurrence of nosebleed.

\section{Conflict of Interest: None}

Source of Support: Nil

\section{References}

1. Pharmacologic Prevention of Migraine: A Narrative Review of the State of the Art in 2018- Elizabeth Loder etal: Headache. 2018

2. Migrainomics identifying brain and genetic markers of migraine Dale R. Nyholt etal :Nature Reviews Neurology vol 13, pages725-741:2017

3. Acute treatment of Migraine -Bert .B .Vergas: Continuum. August 2018 ,vol 24,No 24:1032-1051

4. Migraine and its psychiatric comorbiditiesMinen MT, et al. J Neurol Neurosurg Psychiatry 2016; 87:741-749.

5. Biological insights from the premonitory symptoms of migraine-Nazia Karsan \& Peter J. Goadsby : Nature Reviews Neurology volume 14, pages699710 (2018)

6. Migraine and epistaxis: An interesting combination- Sweta Adatia et al. Neurology Asia 2012; 17(2): 153 - 154.

7. Relationships between Epistaxis, Migraines, and Triggers in Hereditary Hemorrhagic Telangiectasia. Amy Elphick etal -Laryngoscope 124: July 2014.

8. Migraine-Induced Epistaxis - Ami D. Sperber et al - Headache 26:517-518, 1986.

9. Migraine and Recurrent Epistaxis in Children. Imad T. Jarjour etal. Pediatr Neurol 2005; 33:94-97.

10. Epistaxis accompanying migraine attacks. Letter to the editor. E Durán-Ferreras etal. Cephalalgia, 2007, 27, 958-959.

11. Neurobiology of migraine -P. J. Goadsby etal. Neuroscience 161 (2009) 327-341.

12. Epistaxis management - our point of view and literature review-Romanian Journal of Rhinology, Vol. 7, No. 28, October December 2017. 